\title{
'Designer babies' almost thirty years on
}

\author{
Alan H Handyside \\ London Women's Clinic, London and the School of Biosciences, University of Kent, Canterbury, UK
}

Correspondence should be addressed to A H Handyside; Email: alan.handyside@londonwomensclinic.com

This paper is part of an Anniversary Issue celebrating 40 years of in vitro fertilisation. The Guest Editor for this section was Lord Robert Winston.

\begin{abstract}
The first pregnancies and live births following in vitro fertilisation (IVF) and preimplantation genetic testing (PGT), formerly known as preimplantation genetic diagnosis, were reported in 1990, almost 30 years ago, in several couples at risk of $X$-linked inherited conditions, which typically only affect boys inheriting the $X$ chromosome with the affected gene from their carrier mothers. At that time, it was only possible to identify the sex of the embryo by amplifying a Y-linked repeat sequence in single cells biopsied at cleavage stages and avoid the transfer of males, half of which would be affected. The extensive publicity surrounding these cases and the perceived risk of using IVF and PGT for desirable characteristics not related to health, such as sex selection, led to the epithet of 'designer babies' which continues to resonate to this day. Here, I briefly reflect on how the technology of PGT has evolved over the decades and whether it deserves this reputation. With efficient methods for whole genome amplification and the genomic revolution, we now have highly accurate universal tests that combine marker-based diagnosis of almost any monogenic disorder with the detection of aneuploidy. PGT is now clinically well established and is likely to remain a valuable alternative for couples at risk of having affected children.

Reproduction (2018) 156 F75-F79
\end{abstract}

\section{Introduction}

In April, 1990, Lord Robert Winston and I, leading a small group at Hammersmith Hospital in London, published a report in Nature on the first pregnancies following in vitro fertilisation (IVF), embryo biopsy and genetic screening for inherited disease, known then as preimplantation genetic diagnosis (PGD), in several couples at risk of having children with various X-linked conditions (Handyside et al. 1990). The timing of that report a week ahead of the decisive free vote in the House of Commons on the Human Fertilisation and Embryology Bill and whether to allow research on early human embryos was no coincidence. The late Sir John Maddox, then Editor, was adamant that this work, which demonstrated the potential clinical benefits of allowing human embryo research, should be in the public domain to inform the national debate. To meet tight publication deadlines, the paper was submitted, peer reviewed and accepted within days, and to ensure maximum publicity Nature organised a press conference.

The day after the press conference, at which the first two patients now heavily pregnant with twins were present, and publication of the report, PGD made headlines on the front pages of UK national newspapers and around the world. The reception was mixed. While there was support from patients and some clinical geneticists who recognised the trauma of terminating affected pregnancies following conventional prenatal diagnosis of an established pregnancy, some, mainly religious, groups were opposed to PGD in principle and particularly concerned about the destruction of affected human embryos. Others warned of a 'slippery slope' that would eventually allow parents to screen their embryos for particular characteristics such as sex, hair or eye colour or even intelligence. The epithet 'designer baby' was born. Nevertheless, the Bill was subsequently passed by a substantial majority and included provision for PGD for serious conditions and for human embryo research aimed at improving the understanding and diagnosis of inherited disease. Almost 30 years on were the fears about the slippery slope to designer babies justified? Did we get the regulation of PGD right? How successful has PGD been in clinical practice and what does the future hold in the era of the personal genome?

\section{Preimplantation genetic testing of monogenic disease}

PGD involves couples at risk of having children with a single gene defect having IVF treatment, the removal or biopsy of one or more cells from each embryo for genetic analysis and the selection of unaffected embryos for transfer. The two polar bodies, by-products of the two meiotic divisions in the oocyte 
and containing the excess maternal chromosomes, can be biopsied to analyse for meiotic chromosome segregation errors or maternally inherited single gene defects. Later, one or two cells can be biopsied from cleavage stage embryos or multiple trophectoderm cells biopsied at the blastocyst stage. Today, with the availability of highly effective cryopreservation, using rapid cooling and vitrification, most biopsies are performed at the blastocyst stage. The biopsied embryos are then vitrified allowing time for the genetic analysis before thawing unaffected embryos for transfer in a later unstimulated cycle. Recently, the nomenclature has been changed to preimplantation genetic testing (PGT) for monogenic disorders (PGT-M) to distinguish it from preimplantation genetic screening or PGT for chromosome aneuploidy (PGT-A), which is used with infertile patients to avoid the transfer of aneuploid embryos, and PGT for structural chromosome imbalance (PGT-SC) (Zegers-Hochschild et al. 2017). Here, I focus on the development of PGT-M.

Single-cell analysis for PGT-M following cleavage stage biopsy was first used to identify the sex of embryos in a series of couples at risk of various X-linked conditions, which typically only affect males (Handyside et al. 1990). The use of PCR at the singlecell level was still in its infancy and even doubling the typical number of amplification cycles failed to amplify unique target sequences reliably. For this reason, PCR amplification of a Y-linked repeat sequence, present in thousands of copies per cell, was used for identification of male embryos. However, the extreme sensitivity of this protocol and the rapid build-up of amplified DNA products in the laboratory environment, soon led to the appearance of contamination and false-positive results in blank (negative) controls. Furthermore, in a minority of single male cells, amplification still failed, resulting in a male embryo being identified as female, which led to the first clinical misdiagnosis following PGT-M.

The breakthrough, enabling amplification of short unique sequences encompassing the site of the gene mutation, was to use two rounds of PCR amplification, preferably with nested oligonucleotide primers (Coutelle et al. 1989, Holding \& Monk 1989). Using this strategy, PGD of single gene defects using single biopsied cells became possible resulting in the birth of a child free of the common $\Delta \mathrm{F} 508,3$ base pair deletion of the CFTR gene causing cystic fibrosis (Handyside et al. 1992).

Nested PCR of a short fragment of exon 10 of the CFTR gene, including the sequence around the position of $\Delta F 508$, remains one of the most sensitive and efficient methods of single-cell DNA amplification. Nevertheless, in a small percentage of single cells, amplification either fails completely or there is an apparently random failure to amplify one of the two parental alleles, a phenomenon, known as allele dropout (ADO).

\section{Multiplex PCR for PGT-M}

The combined problems of ADO and contamination prompted the search for DNA markers, which could be used to identify independently the presence of the chromosome carrying the mutant gene, essentially producing a diagnosis with built-in redundancy (Ao et al. 1998). In the early 1990s, the intense effort to sequence the human genome led to the discovery and mapping of a highly polymorphic class of markers, known as short tandem repeats (STRs), which are widely distributed throughout the genome and consist of a short sequence of two or more bases repeated a variable number of times. By selecting a combination of STRs in close proximity to the mutation site, having different repeat lengths (i.e. different alleles) on all four parental chromosomes, it is possible to track the inheritance of each chromosome from parent to embryo. Although ADO can potentially affect STR loci as well the mutation sites, the probability of multiple loci on the same chromosome all being affected in the same cell is very low. Until recently, this strategy of using flanking STR markers, combined with mutation detection, has been the 'gold standard' for PGT-M (Fiorentino et al. 2006). The relatively short length of the STRs (no more than a few hundred base pairs), combined with the sensitivity of fluorescent PCR analysed by capillary electrophoresis using automated sequencers, facilitates simultaneous amplification of multiple target sequences (multiplex PCR) directly from single or small numbers of cells. Also, mutation detection can be done simultaneously using single fluorescent base extension reactions, or minisequencing, with specially designed primers.

\section{SNPs and karyomapping}

The problem with multiplex fluorescent PCR is that, although the reagents are relatively low in cost, the initial work to identify informative markers for a specific family or inherited condition and optimise their amplification at the single cell level, is time consuming and labour intensive. Furthermore, it may be difficult to identify fully informative closely linked markers in some families, resulting in the testing of numerous potential candidates and a final combination, which may only be semiinformative or less closely linked to the mutant gene than desired. For this reason, multiplex panels of markers have been developed for preimplantation genetic haplotyping (PGH) of specific inherited conditions. In the PGH approach, the disease-linked STRs used are sufficiently numerous that most families are found to have at least two or three that are informative and therefore able to provide a diagnosis (Renwick et al. 2006). Nonetheless, some couples are still excluded because of lack of informative markers within the STR panels and any new or rare condition still requires the development of an additional single cell test. 
With the development of new methods for whole genome amplification by, for example, the use of isothermal multiple displacement amplification, it is now possible to amplify microgram quantities of DNA from a single cell (Handyside et al. 2004). This ushered in a genomics revolution in single-cell analysis allowing, for example, whole genome sequencing using next generation sequencing (NGS) with a broad range of applications, particularly in the study of tumorigenesis (Fig. 1). For PGT-M, targeted sequencing of hundreds of thousands of SNPs genome-wide on a microarray platform provides a universal set of markers across each chromosome. SNPs are a common type of genetic variation, in which one of two or more bases may be present at a particular position in the DNA sequence. Over ten million SNPs have been identified in the human genome and allele frequencies studied by genotyping multiple individuals in different populations. Biallelic SNPs with a relatively high minor allele frequency and heterozygosity ratio represent an important set of DNA markers. By convention, each SNP locus is genotyped

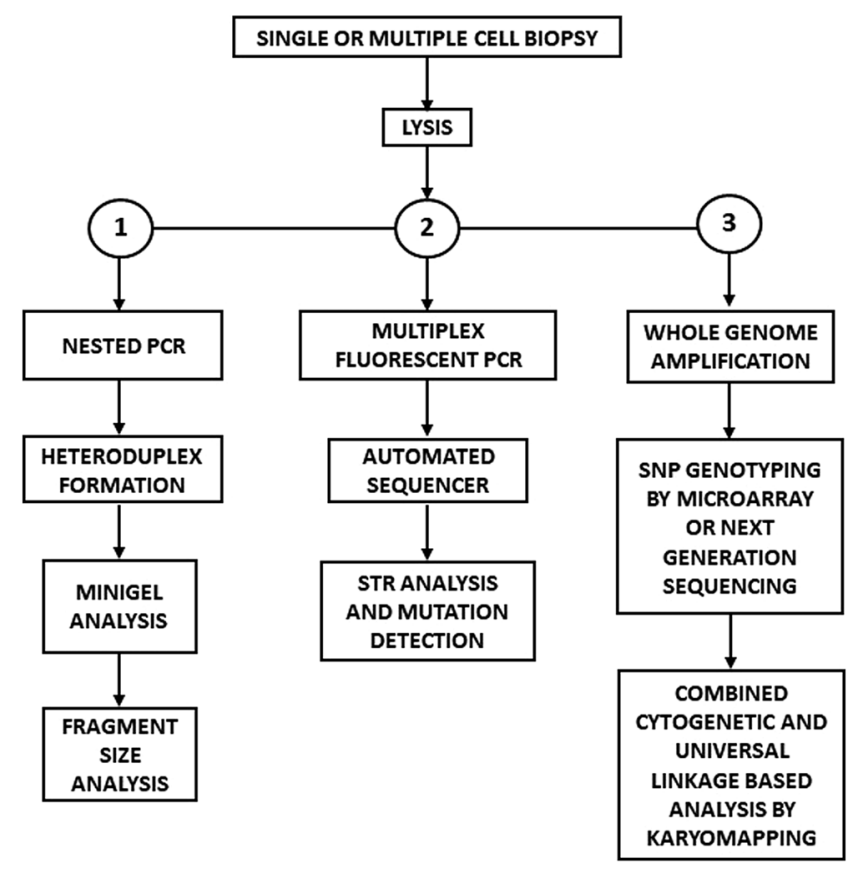

Figure 1 The evolution of methods for preimplantation genetic testing for monogenic disease (PGT-M). (1) The first methods for detecting monogenic disease involved nested PCR to amplify sufficient DNA for rapid analysis, for example, by heteroduplex detection and minigel polyacrylamide gel electrophoresis within a few hours (Handyside et al. 1992). (2) To avoid misdiagnosis caused by ADO, multiplex fluorescent PCR protocols were developed to combine analysis of informative short tandem repeats and mutation detection by minisequening on automated capillary electrophoresis sequencers (Fiorentino et al. 2006). (3) Most recently, whole genome

amplification, genome-wide SNP genotyping by microarrays or next generation sequencing (NGS) and karyomapping provides a universal set of markers for cytogenetics and linkage-based PGT-M (Handyside et al. 2010, Natesan et al. 2014, Zamani Esteki et al. 2015). as $\mathrm{AA}$ or $\mathrm{BB}$ if homozygous, or $\mathrm{AB}$, if heterozygous, irrespective of the actual nucleotides (i.e. G, C, A or T) present.

Biallelic SNP markers, by definition, can only distinguish two out of the four parental chromosomes. However, by determining the genotypes of the parents and working out which of the $A B$ alleles at each position is present on individual chromosomes (phasing) using, for example, an existing child of known disease status, four different sets of genome-wide SNP markers can be identified (Fig. 2). Karyomapping uses a beadarray to genotype 300K SNPs genome-wide, Mendelian analysis and an algorithm to avoid errors caused by ADO (Handyside et al. 2010, Natesan et al. 2014). The main advantage of karyomapping is that the vast majority of even rare monogenic diseases (or combinations of defects) within the regions covered by the SNP markers can be diagnosed accurately in one universal test, based on the SNP markers present closely linked to or within the affected gene(s). Also, because there are markers for each parental chromosome, meiotic trisomies, monosomies and subchromosomal deletions can be identified. Furthermore, extended algorithms have been developed to include both genotype-based analysis and allele-specific quantitation (Johnson et al. 2010, Zamani Esteki et al. 2015). SNP genotyping and karyomapping is therefore a powerful method for analysing all aspects of the genome at the single-cell level, including general features such as 'fingerprinting' transferred embryos and detecting contamination (Table 1). Karyomapping has now become the 'gold standard' for linkage-based PGT-M and combined with analysis of the whole genome amplified DNA by NGS-based chromosome

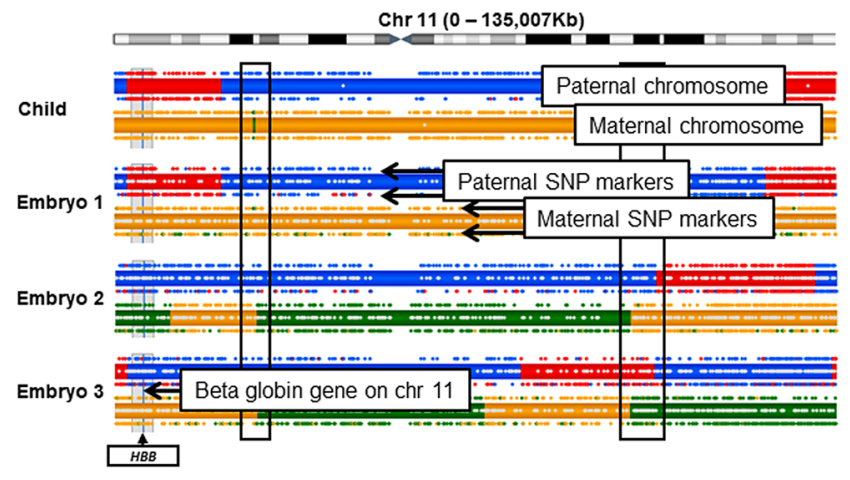

Figure 2 Preimplantation genetic testing for beta thalassaemia by SNP genotyping and karyomapping. Karyomaps of single blastomeres biopsied from cleavage stage embryos. (A) Karyomaps for chromosome 11 in three embryos from a PGD case for $\beta$-thalassaemia and the unaffected child born following transfer of Embryo 1. The sibling used as a reference to phase the SNP calls is a carrier of the affected paternal allele. Note the consistent pattern of key SNPs and non-key SNPs (coloured dots) above and below the predicted haploblocks. Also note the crossovers in both the paternal (upper) and maternal (lower) chromosomes, common to all samples, indicating crossovers in the reference. Modified from Natesan et al. (2014). 
Table 1 Genome-wide analysis by SNP genotyping and karyomapping.

\begin{tabular}{ll}
\hline Genetic feature & Karyomapping \\
\hline Linkage markers & Genome-wide SNP coverage \\
Intragenic markers & Commonly available \\
Meiotic trisomy & Parent of origin and MI or MII type \\
Mitotic trisomy & Extended algorithms only* \\
Monosomy & Parent of origin \\
Deletions (>1-5 Mb) & High resolution/parent of origin \\
Uniparental heterodisomy & Parent of origin \\
Uniparental isodisomy & Extended algorithms only* \\
Triploidy & Parent of origin \\
Tetraploidy & Not detected \\
Single nucleotide variants & Not detected \\
Consanguinity & Informative unless all chr shared \\
Paternity testing & Highly informative \\
Chromosome/embryo & Unique pattern of recombination events \\
fingerprint & \\
Contamination & Detected \\
\hline
\end{tabular}

*Johnson et al. (2010), Zamani Esteki et al. (2015).

copy number analysis to identify non-viable aneuploid embryos has significantly improved pregnancy and live birth outcomes.

\section{The next 30 years}

Following the debates in both Houses of Parliament in the UK in 1990, legislation to allow PGT and embryo research was passed and the Human Fertilisation and Embryology Authority (HFEA) was set up to regulate all aspects of IVF treatment and research. To counteract fears that PGT would be used by parents to have children with desirable characteristics unrelated to health, so called 'designer babies', the HFEA organised a public consultation on the possibility of using PGT to select and transfer embryos of known sex, simply to have a child of a particular sex. At that time, a majority of responses were against allowing sex identification and the HFEA therefore used their regulatory powers to prevent this information being made available, except in cases of X-linked disease. This was subsequently included in amended legislation. Other countries with particular issues related to sex such as Taiwan and India have followed the UK's lead. However, other countries notably the USA and Spain continue to allow PGT for sex identification.

Beyond sex selection, there was considerable debate in the early years around the severity of monogenic diseases for which PGT should be allowed. However, further public consultation on PGT for late-onset conditions such as Huntington's chorea and genetic predisposition to cancers was more favourable and these were allowed. This eventually led to regulation on an ad hoc basis for each condition, and there is now a list of conditions which have been licenced for PGT. This has often led to considerable doubt and delay in treating couples. A Freedom of Information request in
2010 revealed that the only application which had not been allowed was for a deletion on the $Y$ chromosome causing severe oligozoospermia (Handyside 2010). In paternal carriers of Y-linked microdeletions, any boys born following intracytoplasmic sperm microinjection are likely to be infertile themselves. Although infertility is not life threatening, it is ironic that the HFEA should not consider this serious enough for testing. In my experience over the last 30 years, apart from sex selection, I have never had a request for PGT for anything trivial. In my opinion, the parents, who generally know at first hand the effects of a genetic condition, are in the best position to make their own reproductive decisions including the possibility of PGT with appropriate genetic counselling.

Beyond sex selection, the ability to 'design' a baby with one or more desired traits such as hair and eye colour, intelligence, sporting or musical ability etc. by PGT and embryo selection is extremely limited. Despite knowing much more about the genetic basis of these traits and having the technical ability to track their inheritance, the right combination of genes still has to be present in the parents and their embryos at sufficiently high frequency to make it feasible to select an appropriate embryo. Furthermore, many complex traits such as intelligence are multigenic and influenced by the environment and the chance of the right combination of multiple genes decreases logarithmically. However, as we know more about all of the variants associated with some conditions, for example, autism, it may be possible to track the inheritance of the major variants in families with a clear history of the condition.

In 2001, Verlinsky and colleagues reported the first live birth following PGT-M for Fanconi's anaemia combined with human leucocyte antigen (HLA) matching by analysis of HLA loci on the short arm of chromosome 6 (Verlinsky et al. 2001). In these blood-related conditions, the aim of PGT is not only to avoid the monogenic disease but also transfer an HLA-matched embryo, so that at birth, histocompatible cord blood stem cells can be isolated for transplantation to an existing sick child. These so-called 'saviour sibling' cases were highly controversial at the time, particularly as in some cases, PGT was used only for HLA matching in non-inherited spontaneous childhood leukaemias for example. Nevertheless, since then, an increasing number of sick children have had stem cell transplantation and been cured of their disease (Kahraman et al. 2014) and in the UK, the HFEA allows HLA matching on a case-by-case basis.

In late 2017, current and past members of the European Society for Human Reproduction and Embryology (ESHRE) PGD Consortium celebrated 20 years since the founding of the group at the annual meeting in Edinburgh in 1997. The PGD Consortium has diligently collected and published data and pregnancy outcomes on all types of PGT from centres around Europe, USA and Australia among other countries and has provided guidelines on best practice. By 2012, the Consortium 
reported the cumulative results, which included over 5000 live births and low misdiagnosis rates (Harper et al. 2012). Pregnancy rates have remained low and are clearly affected by the proportion of unaffected embryos identified, which is related to the inheritance pattern of the inherited condition. However, with genomic approaches such as karyomapping combined with aneuploidy detection, outcomes per transfer should improve and facilitate transfer of single embryos to avoid the adverse consequences of multiple pregnancies.

Recently, there have been breakthroughs in both gene therapy for beta thalassaemia (Boulad et al. 2018) and in genome editing of human embryos (Ma et al. 2017). Beta thalassaemia is the most prevalent inherited disorder world-wide and a potential cure using autologous blood cells is enormously significant. However, for most other single gene defects affecting multiple cell types throughout the body, gene therapy may only offer a partial cure. The efficiency of genome editing using CRISPR/Cas 9 is yet to be demonstrated definitively and the risk of any off-target editing remains a barrier for clinical application. As monogenic disorders, whatever their inheritance pattern, are present typically in only half of the gametes of the carrier parent, selection of unaffected embryos following PGD is likely to remain the simplest and safest approach to preventing the birth of affected children. For couples, PGD is therefore likely to remain an important alternative to prenatal diagnosis, particularly when combined with aneuploidy testing to identify non-viable genomically unbalanced embryos.

\section{Declaration of interest}

The author was until recently a part time employee of Bluegnome Ltd, Cambridge, UK, a wholly owned subsidiary of Illumina, San Diego, CA, USA which manufactures microarrays and sequencing equipment and reagents for preimplantation genetic testing.

\section{Funding}

This research did not receive any specific grant from any funding agency in the public, commercial or not-for-profit sector.

\section{References}

Ao A, Wells D, Handyside AH, Winston RM \& Delhanty JD 1998 Preimplantation genetic diagnosis of inherited cancer: familial adenomatous polyposis coli. Journal of Assisted Reproduction and Genetics 15 140-144. (https://doi.org/10.1023/A:1023008921386)

Boulad F, Mansilla-Soto J, Cabriolu A, Rivière I \& Sadelain M 2018 Gene therapy and genome editing. Hematology/Oncology Clinics of North America 32 329-342. (https://doi.org/10.1016/j.hoc.2017.11.007)

Coutelle C, Williams C, Handyside A, Hardy K, Winston R \& Williamson R 1989 Genetic analysis of DNA from single human oocytes: a model for preimplantation diagnosis of cystic fibrosis. BMJ 299 22-24. (https://doi. org/10.1136/bmj.299.6690.22)

Fiorentino F, Biricik A, Nuccitelli A, De Palma R, Kahraman S, lacobelli M, Trengia V, Caserta D, Bonu MA, Borini A et al. 2006 Strategies and clinical outcome of 250 cycles of Preimplantation Genetic Diagnosis for single gene disorders. Human Reproduction 21 670-684. (https://doi. org/10.1093/humrep/dei382)

Handyside A 2010 Let parents decide. Nature 464 978-979. (https://doi. org/10.1038/464978a)

Handyside AH, Kontogianni EH, Hardy K \& Winston RM 1990 Pregnancies from biopsied human preimplantation embryos sexed by Y-specific DNA amplification. Nature 344 768-770. (https://doi.org/10.1038/344768a0)

Handyside AH, Lesko JG, Tarín JJ, Winston RML \& Hughes MR 1992 Birth of a normal girl after in vitro fertilization and preimplantation diagnostic testing for cystic fibrosis. New England Journal of Medicine 327 905-909. (https://doi.org/10.1056/NEJM199209243271301)

Handyside AH, Robinson MD, Simpson RJ, Omar MB, Shaw M-A, Grudzinskas JG \& Rutherford A 2004 Isothermal whole genome amplification from single and small numbers of cells: a new era for preimplantation genetic diagnosis of inherited disease. Molecular Human Reproduction 10 767-772. (https://doi.org/10.1093/molehr/gah101)

Handyside AH, Harton GL, Mariani B, Thornhill AR, Affara N, Shaw MA \& Griffin DK 2010 Karyomapping: a universal method for genome wide analysis of genetic disease based on mapping crossovers between parental haplotypes. Journal of Medical Genetics 47 651-658. (https:// doi.org/10.1136/jmg.2009.069971)

Harper JC, Wilton L, Traeger-Synodinos J, Goossens V, Moutou C, SenGupta SB, Pehlivan Budak T, Renwick P, De Rycke M, Geraedts JPM et al. 2012 The ESHRE PGD Consortium: 10 years of data collection. Human Reproduction Update 18 234-247. (https://doi.org/10.1093/humupd/dmr052)

Holding C \& Monk M 1989 Diagnosis of beta-thalassaemia by DNA amplification in single blastomeres from mouse preimplantation embryos. Lancet 2 532-535. (https://doi.org/10.1016/S0140-6736(89)90655-7)

Johnson DS, Gemelos G, Baner J, Ryan A, Cinnioglu C, Banjevic M, Ross R, Alper M, Barrett B, Frederick J et al. 2010 Preclinical validation of a microarray method for full molecular karyotyping of blastomeres in a 24-h protocol. Human Reproduction 25 1066-1075. (https://doi. org/10.1093/humrep/dep452)

Kahraman S, Beyazyurek C, Yesilipek MA, Ozturk G, Ertem M, Anak S, Kansoy S, Aksoylar S, Kuşkonmaz B, Oniz H et al. 2014 Successful haematopoietic stem cell transplantation in 44 children from healthy siblings conceived after preimplantation HLA matching. Reproductive Biomedicine Online 29 340-351. (https://doi.org/10.1016/j.rbmo.2014.05.010)

Ma H, Marti-Gutierrez N, Park S-W, Wu J, Lee Y, Suzuki K, Koski A, Ji D, Hayama T, Ahmed R et al. 2017 Correction of a pathogenic gene mutation in human embryos. Nature 548 413-419. (https://doi. org/10.1038/nature23305)

Natesan SA, Bladon AJ, Coskun S, Qubbaj W, Prates R, Munne S, Coonen E, Dreesen JCFM, Stevens SJC, Paulussen ADC et al. 2014 Genome-wide karyomapping accurately identifies the inheritance of single-gene defects in human preimplantation embryos in vitro. Genetics in Medicine 16 838-845. (https://doi.org/10.1038/gim.2014.45)

Renwick PJ, Trussler J, Ostad-Saffari E, Fassihi H, Black C, Braude P, Ogilvie CM \& Abbs S 2006 Proof of principle and first cases using preimplantation genetic haplotyping - a paradigm shift for embryo diagnosis. Reproductive Biomedicine Online 13 110-119. (https://doi. org/10.1016/S1472-6483(10)62024-X)

Verlinsky Y, Rechitsky S, Schoolcraft W, Strom C \& Kuliev A 2001 Preimplantation diagnosis for Fanconi anemia combined with HLA matching. JAMA 285 3130-3133. (https://doi.org/10.1001/jama.285.24.3130)

Zamani Esteki M, Dimitriadou E, Mateiu L, Melotte C, Van der Aa N, Kumar P, Das R, Theunis K, Cheng J, Legius E et al. 2015 Concurrent whole-genome haplotyping and copy-number profiling of single cells. American Journal of Human Genetics 96 894-912. (https://doi.org/10.1016/j.ajhg.2015.04.011)

Zegers-Hochschild F, Adamson GD, Dyer S, Racowsky C, de Mouzon J, Sokol R, Rienzi L, Sunde A, Schmidt L, Cooke ID et al. 2017 The International Glossary on Infertility and Fertility Care, 2017. Fertility and Sterility 108 393-406. (https://doi.org/10.1016/j.fertnstert.2017.06.005)

Received 25 March 2018

First decision 27 April 2018

Revised manuscript received 19 May 2018

Accepted 12 June 2018 